\title{
EL PENSAMIENTO DE KANT EN ALGUNAS TEORÍAS RECIENTES DE JUSTICIA SOCIAL
}

María Julia Bertomeu*

SÍNTESE - Neste artigo, a autora aborda a questão da possibilidade de uma teoria da justiça social que seja compativel com a totalidade do sistema kantiano, examinando diversas posições acerca dessa problemática, seguindo-se a essa parte a exposição de sua própria posição no tocante a essa questão.

PALAVRAS-CHAVE - Kant. Justiça social. Razão prática.

\begin{abstract}
In this paper, the author examines the possibility of our having a theory of social justice which is compatible with the Kantian system as a whole. She discusses several competing views and puts forward her own proposal about the problem.
\end{abstract}

KEY WORDS - Kant. Social justice. Practical reason.

Pensar en las características de una teoría de justicia social que sea compatible con la totalidad del sistema kantiano implica una ardua tarea reconstructiva que en los últimos años ha sido realizada, entre otros, por autores de la talla de John Rawls y de J. Habermas. Creo que hay suficiente complejidad y riqueza en la propuesta kantiana como para embarcarse una vez más en reconstruir sus ideas y aportar algo al debate inconcluso, dentro del cual o bien se lo ha ubicado a Kant en una posición muy cercana a una propuesta neoliberal de justicia social fundada en un concepto negativo y restrictivo de libertad externa, o se lo ha reconocido como un auténtico precursor de un estado liberal igualitarista o por último, se lo ha registrado como uno de los antecesores de una teoría republicana de justicia. En la primera parte del trabajo pretendo mostrar quiénes y de que modo han sostenido las posiciones mencionadas. En la segunda parte trataré de esbozar sintéticamente mi propia posición.

I. El pensamiento político de Kant referido a temas de justicia ha sido desarrollado, entre otros, en su trabajo sobre los "Principios metafísicos del derecho" y en

* Universidad Nacional de La Plata y CONICET (Consejo de Investigaciones Cientificas y Tecnológicas - Buenos Aires).

\begin{tabular}{|l|l|l|l|l|l|}
\hline VERITAS & Porto Alegre & v. 45 & n. 4 & Dezembro 2000 & p. 573-582 \\
\hline
\end{tabular}


los Escritos sobre filosofía de la historia y política. ${ }^{1}$ Allí Kant elaboró conceptos tales como "el principio universal del derecho", el "postulado jurídico de la razón práctica", "la libertad como derecho innato único", "el contrato" y "la república". De acuerdo con la prioridad que se esté dispuesto a otorgar a cada uno de ellos, como a las conexiones conceptuales que se admitan o rechacen entre la moral, el derecho y la política kantianas, pueden surgir interpretaciones disímiles y en algunos casos contrarias sobre el problema de la justicia social. Comenzaré con las interpretaciones minimalistas.

I. La exposición del "principio universal del derecho" y de la libertad como derecho único y originario que le corresponde a los hombres en virtud de su humanidad, temas que Kant desarrolló en la "Introducción" a la Metafísica de las costumbres, han sido considerados por algunos intérpretes como los puntos básicos que permiten reconstruir la concepción kantiana de la justicia social, dado que aquí Kant intentó limitar la esfera de la ética política mediante una teoria de la coacción legítima por parte del Estado. El principio universal del derecho fija los límites de toda coacción y prescribe que "una acción es conforme a derecho cuando ella o su máxima permiten a la libertad del arbitrio de cada uno coexistir con la libertad de todos según una ley universal." ${ }^{2}$ De acuerdo a esta ley fundamental que sólo afecta al aspecto externo de las acciones, la coacción por parte del estado es legítima frente a los actos que obstaculizan o interfieren a la libertad según leyes universales. ${ }^{3}$ Por otro lado Kant considera que la libertad o sea, "la independencia con respecto al arbitrio constrictivo de todo otro compatible con la libertad de cualquiera según una ley universal", es el derecho único y originario. Ahora bien, si tomamos, por ejemplo, la interpretación que en el ámbito anglosajón ha propuesto Jeffrie G. Murphy ${ }^{4}$ para plantear el tema de la justicia distributiva en Kant, veremos que ciñéndose casi exclusivamente a estos dos puntos, Murphy se pregunta en qué sentido, coherente con la teoría kantiana, sería posible pensar que el gobierno tiene el derecho y la obligación de implementar impuestos para contribuir al bien general, dado que su función propia consiste en evitar las interferencias a la libertad en nombre de la misma libertad, cosa que se logra ejerciendo el poder sobre quienes lesionan derechos perfectos para con los demás. El argumento de Murphy es que, en este contexto kantiano, si bien es bueno moralmente ayudar a los demás, sin embargo no es obligatorio en el sentido fuerte que requiere una justificación de la coerción por parte del Estado, dado que la intervención coactiva se justifica exclusivamente cuando la libertad ha sido violada. La solución que propone Murphy es reinterpretar a la libertad en términos más amplios pero igualmente negativos, recordando que ciertos tipos de arreglos econó-

1 Kants Werke, Reproducción fotomecánica del texto de la Prussischen Akademie der Wissenschaften de 1902 Berlín, Walter de Gruyter, Band VIII, Abhandlungen nach 1781, Band VI: Die Metaphysik der Sitten.

2 I. Kant, Metaphysik der Sitten, op.cit, Einleitung, C, 230.

3 I. Kant, Metaphysik der Sitten, op.cit, Einleitung, . D, 231.

4 J. Murphy, Kant, The Philosophy of Right, Macmillan, London, 1970, p. 146. 
micos y sociales inhiben, en un sentido literal, a la libertad de aquellos que están en desventaja. No me interesa concentrar la atención en la solución que plantea Murphy, sino en el modo en que presenta el problema. Sin duda alguna, el Kant que Murphy recorta está muy cercano a un planteo liberal de mínimos. En primer lugar porque la libertad que se está tutelando mediante la ley universal del derecho es una libertad frente a las interferencias deliberadas por parte de otros seres humanos - libertad negativa en el sentido que le dio Isaiah Berlin - que sería compatible con un estado en el cual todos estuvieran ocupados solamente por sus intereses privados. En efecto, según Kant, "cada uno puede ser libre, aunque su libertad me resulte totalmente indiferente o desee cordialmente dañarla, con tal de que no le perjudique con mi acción externa". ${ }^{5}$ Esta libertad externa puede ser realizada independientemente de la relación solidaria entre los ciudadanos y es consistente con la indiferencia por parte de los otros acerca de las necesidades e intereses del prójimo. Plantear el tema de la justicia distributiva en estos términos implica en primer lugar pensar que la disposición o la actitud de los individuos no juega ningún papel en la conformación de una sociedad justa, que los fines no tienen relación con los resultados externos de las acciones, que en el proceso de constitución de las normas no operan factores que también tienen que ver con la realización de la libertad entendida como autolegislación ciudadana, cosa que, según creo, está lejos de ser la propuesta de Kant. El contexto en el cual Murphy plantea el problema de la justicia social en Kant es, a mi modo de ver, demasiado estrecho.

II. Ahora bien, es sabido que Kant establece conexiones conceptuales entre el derecho y la razón pura práctica, entra la política y la moral, de modo tal que resulta plausible pensar que es este último el contexto en el cual deberían ser planteadas las cuestiones de justicia social o que, al menos este contexto es tan importante como el estrictamente político y jurídico. De acuerdo con este tipo de interpretaciones, la teoría kantiana podría dar origen a una concepción igualitaria de justicia social que justifique un estado redistributivo, derechos sociales o bienes primarios. A esta altura resulta casi redundante recordar el papel fundador que ha tenido en esta línea interpretativa la obra de John Rawls. ${ }^{6}$ La persona moral que constituye la noción medular de la concepción de la justicia como imparcialidad de Rawls absorbe los rasgos sobresalientes del sujeto moral kantiano. En efecto, los ciudadanos de la sociedad bien ordenada son autónomos tanto porque eligen y llevan a cabo sus planes de vida, como porque son capaces de limitar sus intereses frente a los principios que emanan de la voluntad de todos ${ }^{7}$. Esta idea ha sido asociada en reiteradas ocasiones por Rawls con el sujeto autolegislador kantiano.

I. Kant, Metaphysik der Sitten, op.cit. C, 231.

6 J. Rawls, "Kantian constructivism in moral theory", Journal of Philosophy, Vol LXXVII, n 9, septiembre 1980, pp.515-573.

7 Para este punto ver M. J. Bertomeu y Graciela Vidiella, "Persona moral y justicia distributiva", Agora 14, II Santiago de Compostela. 
Por otro lado Rawls también ha revalorizado el aspecto de la persona considerada como un fin en sí misma, al punto que llegó a pensar que sus dos principios de justicia ofrecen una interpretación, desde el punto de vista social, del mandato contenido en la segunda formulación del imperativo categórico. Sin duda alguna que la formulación del imperativo categórico kantiano contiene una riqueza incalculable cuando se trata de fundamentar criterios para la distribución de recursos sociales que superen una noción minimalista sobre aquello que debe distribuir un estado justo. Al considerar a la humanidad como una categoría moral y reclamar que las acciones de los hombres no deben nunca tomar a la persona sólo como un medio sino como un fin en sí mismo, Kant estaba legándonos una herencia que Herman Cohen primero y J. Rawls más tarde, han impulsado de modo manifiesto. Pero volvamos ahora a Kant. ¿De qué modo es posible anudar una propuesta como la rawlsiana con los textos de Kant? ¿Cómo integrar en sus textos a la filosofía política con el imperativo categórico?. Hay varios modos de lograrlo. Uno de ellos, el que emprende Rawls, consiste en utilizar un concepto normativo moral, el de persona, para fundamentar una teoría de justicia como equidad. Dado que este concepto fue articulado por Kant en relación con la idea de fin en sí mismo, la persona autónoma y legisladora se constituye en la piedra de toque de un sistema de justicia distributiva.

Ahora bien, a pesar de que la intención rawlsiana no es la de reconstruir el texto de Kant, su propuesta, según creo, podría explicarse en la siguiente terminología kantiana. El derecho entendido como la "legitimidad de la coacción por parte del estado" es independiente de la ética, sin embargo, al referirse al derecho externo estricto, a aquel que no está mezclado con prescripciones referidas a la virtud ni recurre a la conciencia moral como móvil, Kant admitió también un fundamento de otro tipo, centrado en la "conciencia de la obligación de cada uno según una ley", ${ }^{8}$ conciencia que está fundada en el reconocimiento de la razón pura práctica y en el derecho de la humanidad como condición de todos los deberes y fundamento de las obligaciones. ${ }^{9}$ De este modo, el concepto de humanidad o el de derecho de la humanidad en mi propia persona pasa a constituirse también en una fuente de obligación que limita a la libertad exterior y obliga a las personas a abstenerse de tratar a los demás como meros medios. El concepto de humanidad, entendido como "la facultad de la libertad que es totalmente suprasensible y libre de determinaciones físicas", podrá y deberá, ha dicho Kant, ser representado en la doctrina de los deberes tanto jurídicos como morales. ${ }^{10}$ Desde aquí podríamos realizar una inferencia similar a la rawlsiana, pues si conectáramos el derecho de la humanidad en nuestra persona con el concepto de libertad, como el mismo Kant lo hace, esto nos permitiría fundar una concepción de la justicia distributiva en

I. Kant, Metaphysik der Sitten,op.cit., Einleitung, E,232.

9 I. Kant, Metaphysik der Sitten, op.cit. Einleitung, "Einteilung der Metaphysik der Sitten überhaupt", II, 239,20 .

10 I. Kant, Metaphysik der Sitten, op.cit., Einleitung, "Einteilung der Metaphysik der Sitten überhaupt", II. 
cualquiera de las formulaciones del imperativo categórico, aún cuando la segunda formulación es sin duda la más adecuada para tal fin. Si la esencia de la libertad consiste en obrar por respeto a la ley moral, lo cual es equivalente a sostener la autonomía del sujeto, entonces también la autonomía.y no solamente su libertad externa se convierte en piedra de toque de una teoría de justicia política y de los deberes jurídicos .La autonomía moral como garante de una teoría de justicia, por otro lado, es una buena vía de entrada para admitir bienes sociales básicos o el reconocimiento de derechos indispensables para su ejercicio. Ya no se manejaría en este caso un concepto de libertad externa como no interferencia, sino que la autonomía daría lugar a un concepto positivo de libertad y este, a su vez, serviría de sustento para reclamos sociales legitimos.

Pero este tipo de interpretación también podría estar sustentada apelando a los derechos innatos que forman parte del derecho natural fundado en principios a priori y a los cuales Kant, en algunos textos, les ha otorgado el carácter de "fuentes de apelación" para aquellos casos en los cuales se presenta una disputa acerca del derecho adquirido. ${ }^{11} \mathrm{Si}$ bien es cierto que el único derecho innato que les corresponde a los hombres en virtud de su humanidad es el de la libertad, ella es asimismo la fuente de la igualdad de los ciudadanos en cuanto autolegisladores y de la dependencia de todos respecto de una única legislación común como súbditos. También esta podría ser una vía adecuada para fundar una teoría de justicia social igualitaria, aunque con el peligro de una recaída en el derecho natural.

III. Hasta ahora me he limitado a señalar, dentro de la teoría kantiana, los textos que podrían dar lugar a una lectura libertaria o liberal de su ética política. Pero como Habermas ha dicho, quizás no sin razón, se trata de una disputa entre liberales que difieren sobre si la autonomía privada se alcanza mediante derechos civiles o mediante derechos sociales de prestación. En este marco asistimos, según Habermas, a una discusión interna sobre los paradigmas del derecho que está comprometida con un modelo de sociedad productivista para la cual las expectativas de justicia social se satisfacen por medio de individuos que autónomamente (en el sentido de autonomía privada) llevan a cabo sus propias concepciones de la buena vida. ${ }^{12}$ Frente a estos dos modelos que decididamente no comparte, Habermas ha propuesto una complementariedad entre el derecho y la moral, la autonomía privada y la pública, la soberanía popular y los derechos, de modo tal que las libertades del sujeto de derecho privado y la autonomía pública del ciudadano se sustenten de manera recíproca. Para ello ha retomado parcialmente a Kant para reivindicar una lectura procedimental de la tradición republicana. La propuesta de

"Ver para este punto, "Kant, el contrato social y la República: El momento ético de lo político" en O. Guariglia, M. J. Bertomeu.y G.Vidiella, Democracia y estado de Bienestar, Buenos Aires, Centro Editor de América Latina, 1993.

${ }_{12}$ Vease J. Habermas, Die Einbeziehung des Anderen, Frankfurt, Suhrkamp Verlag, 1996, traducción española.

La inclusión del otro. Estudios de Teoria política, a cargo de Juan Carlos Velasco Arroyo y Gerard Vilar Roca, Barcelona, Paidós Ibérica,1999, p. 256. 
Habermas está fundada en los principios básicos de la ética del discurso y en la formación democrática de una opinión y voluntad comunes. El proceso deliberativo, el estado de derecho, el ciudadano como actor colectivo y los actores individuales como variables dependientes son algunos de los temas que le han permitido proponer una complementariedad entre los derechos humanos y la soberanía popular. Los destinatarios del derecho son los autores del mismo, la autonomía privada y pública son mutuamente complementarias o, lo que es lo mismo, el sistema de los derechos está en conexión íntima con la autonomía política de los ciudadanos. Nuevamente aclaro que no me interesa aquí desarrollar la posición de Habermas, sino la presentación que hace de la doctrina kantiana para apoyar, aunque sea de modo parcial, su propuesta.

Es evidente que Habermas intentó recuperar al Kant republicano. Para ello ha puesto en un primer plano a la idea de contrato o de soberanía popular, entendido como una voluntad legislativa común realizada por los ciudadanos en una práctica de autolegislación. Si bien Kant, según Habermas, aceptó que el modelo del derecho en general son las reglas del derecho privado burgués, basado en la libertad de contrato y la propiedad, sin embargo estableció una conexión entre libertad y coacción que permitiría pensar, entre otras cosas, que la pretensión de legitimidad de un orden jurídico construido sobre derechos subjetivos sólo puede resolverse mediante la capacidad de integración social aneja a la voluntad concordante y reunida de todos los ciudadanos libres e iguales. Habermas rastrea y encuentra esta idea en Kant, dado que ese "principio democrático" viene a llenar un vacío en un sistema de egoísmo jurídicamente ordenado. Y puesto que este "vacío de solidaridad" no puede llenarse mediante el recurso a nuevos derechos, es la autonomía de los ciudadanos la que opera como fuerza de integración social. ${ }^{13}$ Volvamos a Kant. La noción de contrato social juega un papel decisivo en la teoría política de Kant, al punto que, en algunos de sus textos, el filósofo sostiene que es el único pilar sobre el cual es posible obtener una constitución civil jurídicamente legítima. Se trata de una idea de la razón que "obliga a cada legislador para que dicte sus leyes tal y como si éstas pudiesen haber nacido de la voluntad reunida de todo un pueblo, y para que considere a cada súbdito en cuanto ciudadano, como si hubiera estado de acuerdo con una voluntad tal". ${ }^{14}$ El contrato aparece como una regla tanto para el legislador como para los ciudadanos, cuando ellos quieren comprobar la legitimidad de la ley. El aspecto novedoso de la propuesta kantiana es que el filósofo enfáticamente subraya que este acuerdo es independiente y absolutamente libre de todo interés particular de las partes. Por otro lado para Kant la idea del contrato posee el carácter de un experimento mental, como el imperativo categórico, que realizan tanto el legislador como los ciudadanos para

${ }^{13}$ J. Habemas, Faktizität und Geltung. Beiträge zur Diskurstheorie des Rechts und des demokratischen Rechstaats. Frankfurt, Suhrkamp Verlag, 1992 y 1994, traducción española Facticidad y validez. Sobre el derecho y el Estado democrático de derecho en términos de teoria del discurso. A cargo de Manuel Jiménez Redondo, Madrid, Trottạ, 1998, p. 94 y 95.

${ }^{14}$ I. Kants Werke Band VII, "Uber die Gemeinspruch...", p. 297. 
averiguar si el sistema jurídico que los coacciona es justo. También ha señalado Kant que no caben cálculos pragmáticos sobre la felicidad o el bienestar de los súbditos porque ninguna ley puede depender de semejante contingencia. Es este uno de los aspectos que, a mi modo de ver, más aleja a Kant de la propuesta de Habermas y más lo acerca a Rawls, al menos en los textos a los que estamos haciendo referencia. El carácter ficticio del contrato kantiano tiene poco en común con un procedimiento democrático destinado a alcanzar acuerdos reales, porque el criterio no es lo que el pueblo realmente dice sino lo que hipotéticamente pudiera provenir de la voluntad reunida del mismo. En este sentido, como bien se ha señalado, Kant produjo una desactivación del potencial revolucionario y de transformación social que encerraba la idea de contrato en Rousseau ${ }^{15}$ y que, en definitiva es la que Habermas pareciera estar reivindicando. El carácter de experimento mental le quita el elemento activo de construcción de una intersubjetividad y formación de voluntad común, que es el soporte de la autonomía política de los ciudadanos en la propuesta de Habermas. Con ello el contrato de Kant pierde la capacidad de integración social que Habermas le atribuye para compensar la debilidad motivacional de la moral racional. Pero al mismo tiempo la pierde porque nunca podría ser pensado como un modo de equilibrar los imperativos funcionales que provienen del mercado y del aparato estatal con los procesos de entendimiento y la búsqueda de solidaridad.

\section{Conclusión}

Hace ya varios años publiqué un trabajo sobre el republicanismo kantiano ${ }^{16}$ en el cual trataba algunos de los temas que he discutido aquí. Sigo pensando que en la filosofía política de Kant juegan un papel importante y muchas veces indelimitable los derechos innatos y el contrato social originario. En la mayoría de sus obras Kant ha señalado que la libertad es el derecho único originario que le corresponde a todo hombre en virtud de su humanidad. En su "Teoría del derecho" esa exclusividad se explica porque es condición de posibilidad de la existencia de todo derecho. El papel regulativo que como derecho innato desempeña aquí la libertad, es similar a aquel que en otros textos que he mencionado anteriormente juega también el contrato social. Creo que Kant no resolvió la tensión entre soberanía popular y derechos innatos, y que en este sentido son plausibles distintas interpretaciones de su propuesta, algunas más cercanas al liberalismo social y otras al republicanismo. No creo, en cambio, que asista razón a la interpretación kantiana en términos liberales mínimos. Discrepo fundamentalmente con aquellas lecturas libertarias de su pensamiento político que acentúan indiscriminadamente el papel de la "propiedad privada" en la constitución de la libertad, en desmedro de la función constitutiva que también ejercen sobre la misma la autolegislación

\footnotetext{
15 Carracedo, José, “El influjo de Rousseau en la filosofía práctica” en E. Guizán (edit.) Esplendor y miseria de la ética kantiana, Barcelona, Anthropos, 1988, pp. 29-74.

16 O.Guariglia, M.J.Bertomeu y G. Vidiella, Democracia y estado de bienestar, op.cit. pp. 43-59.
} 
como modo de expresión de la soberanía popular. Una de las estrategias para lograrlo ha consistido en sobredimensionar algunos pasajes de su obra, en los cuales Kant hace referencia a las características de un "ciudadano activo" o legislador pleno. Efectivamente según Kant, un ciudadano debe poseer ciertas cualidades naturales, no ser niño ni mujer, y otras sociales, ser el señor de sí mismo y, por lo tanto, tener alguna propiedad, incluyendo dentro de las mismas a las habilidades, los oficios, las artes y las ciencias, y excluyendo a quienes deben ganarse la vida por medio de la venta de lo que es suyo. ${ }^{17}$ De estos textos algunos autores han derivado de modo directo una adhesión de Kant a un planteo burgués que no reconoce otra libertad verdadera que la de los propietarios. A mi modo de ver esta interpretación se deriva de una lectura demasiado estrecha del concepto de libertad negativa entendida como ausencia de interferencia. Pero si pensáramos a este concepto con categorías más amplias, sería legítimo correr los límites de la coacción del estado para incluir dentro de su esfera tanto las interferencias contra la propiedad, como contra el ejercicio de la autonomía política o de la ciudadanía activa. Si esto fuera así, el concepto formal de libertad tendría otras repercusiones en la política social distributiva dentro del estado, y permitiría pensar en la distribución de bienes en nombre de esa libertad negativa que justifica la coacción. No encuentro en los textos kantianos razones fundadas para negar esta última posibilidad.

Por otro lado, pienso efectivamente que en la filosofía política kantiana hay una tensión entre la "metafísica del derecho" y "la realización del derecho en la historia". ${ }^{18}$ Pero creo que se ha hecho un uso algo exagerado de algunos de los textos kantianos en los cuales el filósofo habla de la historia como una realización accidentada y fortuita de un estado justo. Me refiero, por ejemplo a los "demonios inteligentes" o de los antagonismos de la sociedad de los cuales se sirve la naturaleza para desarrollar las disposiciones naturales de la humanidad e instaurar una constitución civil justa. ${ }^{19}$ En otros textos Kant habla de una realización histórica distinta, a través de un "fin totalizador" para las máximas universales, e incluso de una unificación de las máximas en un proyecto que estaría tutelado por el concepto de bien supremo. ${ }^{20}$ No tengo espacio para explayarme sobre este tema pero simplemente quiero recordar que tanto en la Crítica del Juicio como en la Religión

17. Ver aquí, Kants Werke, Band VIII, op. cit. pp. 295.

${ }_{18}$ Ver, en este punto, Wolfgang Kersting, "Politics, freedom, and order: Kant's political philosophy" en Paul Guyer (edit.) The Cambridge companion to Kant, Cambridge, New York, Melbourne, Cambridge University Press, 1992, pp. 342-367.

19 Ver aquí, Kants Werke, op. cit.

${ }^{20}$. Pocos autores han resaltado este punto. Entre los de lengua castellana cabe destacar a Antoni Domenech, De la ética a la política, Barcelona, Crítica,1989, G.Vilar, La razón insatisfecha, Barcelona,Crítica, 1999 y mi propia tesis de doctorado, Recepción y vigencia de la ética kantiana a fines del siglo XIX y principios del XX, Universidad Nacional de La Plata, inédita. En otros idiomas, Y.Yovel "The Highest good and history in Kant 's Tought" en Archiv für Geschichte der Philosophie 54, Heft 3, 1972 y G.Krämling, "Das höchste Gut als mögliche Welt", Kant Studien 77, Heft 3, 1986. 
dentro de los límites de la mera razón, ${ }^{21}$ Kant trabajó con una idea de bien supremo que contempla la felicidad de todos en un estado ético político justo, y no requiere una satisfacción inmediata de los deseos individuales sino que se proyecta a las generaciones futuras y a la especie y supone la creación de instancias jurídicas y sociales y el desarrollo de la cultura. Dicha cultura prepara el advenimiento de un estado de dominio de la razón práctica, supone la existencia de ciertas instituciones jurídico-políticas y la disciplina de los instintos. Se trata, sin duda, también de un proceso histórico pero de cooperación recíproca y que supone una automodelación del carácter y no una mera suma de intereses. Ideas similares están contenidas en el Libro III de la Religión cuando Kant habla del bien supremo como fin comunitario que se realiza por medio de la "cooperación" de todos obrando según leyes de virtud.

Por último, creo que para delinear una respuesta a la cuestión planteada, esto es, qué tipo de justicia social sería posible articular a partir de los textos kantianos, es necesario, como ya he dicho, trabajar el concepto kantiano de libertad en los escritos ético políticos. Sin duda alguna este concepto no es equivalente al de libertad positiva como autonomía moral, pues de serlo el derecho y la política estarían absolutamente subordinados a la moral. Sin embargo, el concepto de libertad que Kant piensa en relación con el pueblo todo, en el cual todos deciden sobre todo, aunque respetando los mecanismos legítimos de producción de las normas, haría pensar en la existencia de ciertas capacidades, o al menos en la ausencia de ciertas vulnerabilidades, que irían mucho más lejos que una mera concepción de libertad como no interferencia actual y real. Y si esto es así, este concepto negativo de libertad daría lugar al reconocimiento de derechos sociales y políticos de un amplio espectro y a un diseño de las instituciones sociales distinto de una mera suma de intereses particulares. Si el proyecto político republicano de Kant es el de la libertad ante la ley, si en este proyecto se fundamenta la posibilidad de coacción por parte del Estado, y si la autolegislación política de los ciudadanos es la fuente de legitimidad de la misma, entonces nuevamente será indagando a la libertad que llegaremos a una solución del problema de la justicia.

${ }^{21}$ Kants Werke, op.,cit Band V Kritik der Urteilskraft, especialmente par. 83-84 y VI Die Religion innerhalb der Grenzen der blossen Vernunft, especialmente Libro III. 